\title{
Improvement of an Escherichia coli whole-cell biocatalyst for geranyl glucoside production using directed evolution
}

\author{
Julian Ruediger ${ }^{1}$ and Wilfried Schwab ${ }^{1}$ \\ ${ }^{1}$ Technical University of Munich
}

March 10, 2021

\begin{abstract}
The biotechnological production of glycosides is an economically competitive manufacturing alternative to classical chemical synthesis. Through continuous production improvement, glycosides can now be used in low-cost products by various industries. However, many production systems still suffer from low yields. Directed evolution, coupled with a suitable screening method, can tackle this challenge. We generated glycosyltransferase mutants through error-prone-PCR and screened the library using a small-scale whole-cell biotransformation system. The screening of only 176 colonies yielded three putative candidates. Detailed investigations revealed that the reason for the increase in product titer was mainly due to different expression effects of the mutagenized genes rather than improved enzyme kinetics. In total, a 60-fold increase in product formation was achieved. Therefore, in addition to the quality of the mutant library, an efficient and stable expression system is crucial to achieve high concentrations of active enzyme and product, as formation of inclusion bodies and other inactive forms of the biocatalyst reduces productivity.
\end{abstract}

\section{Hosted file}

SCREENING manuscript JR_WS.pdf available at https://authorea.com/users/400642/articles/ 512942-improvement-of-an-escherichia-coli-whole-cell-biocatalyst-for-geranyl-glucosideproduction-using-directed-evolution

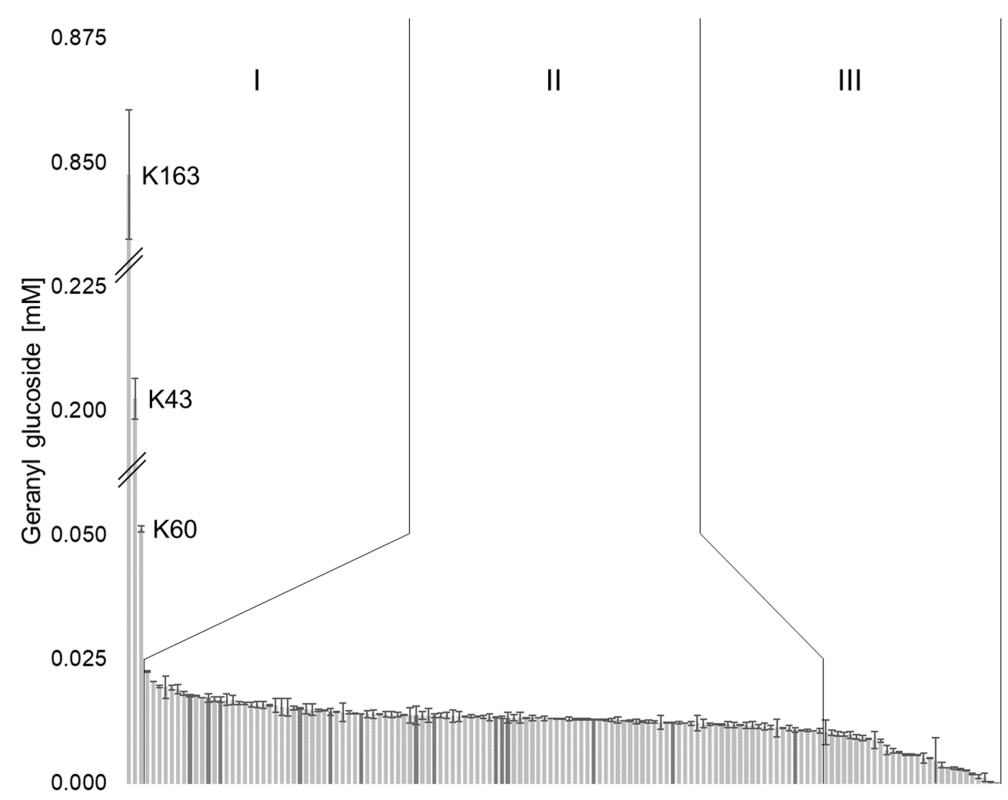

IV 

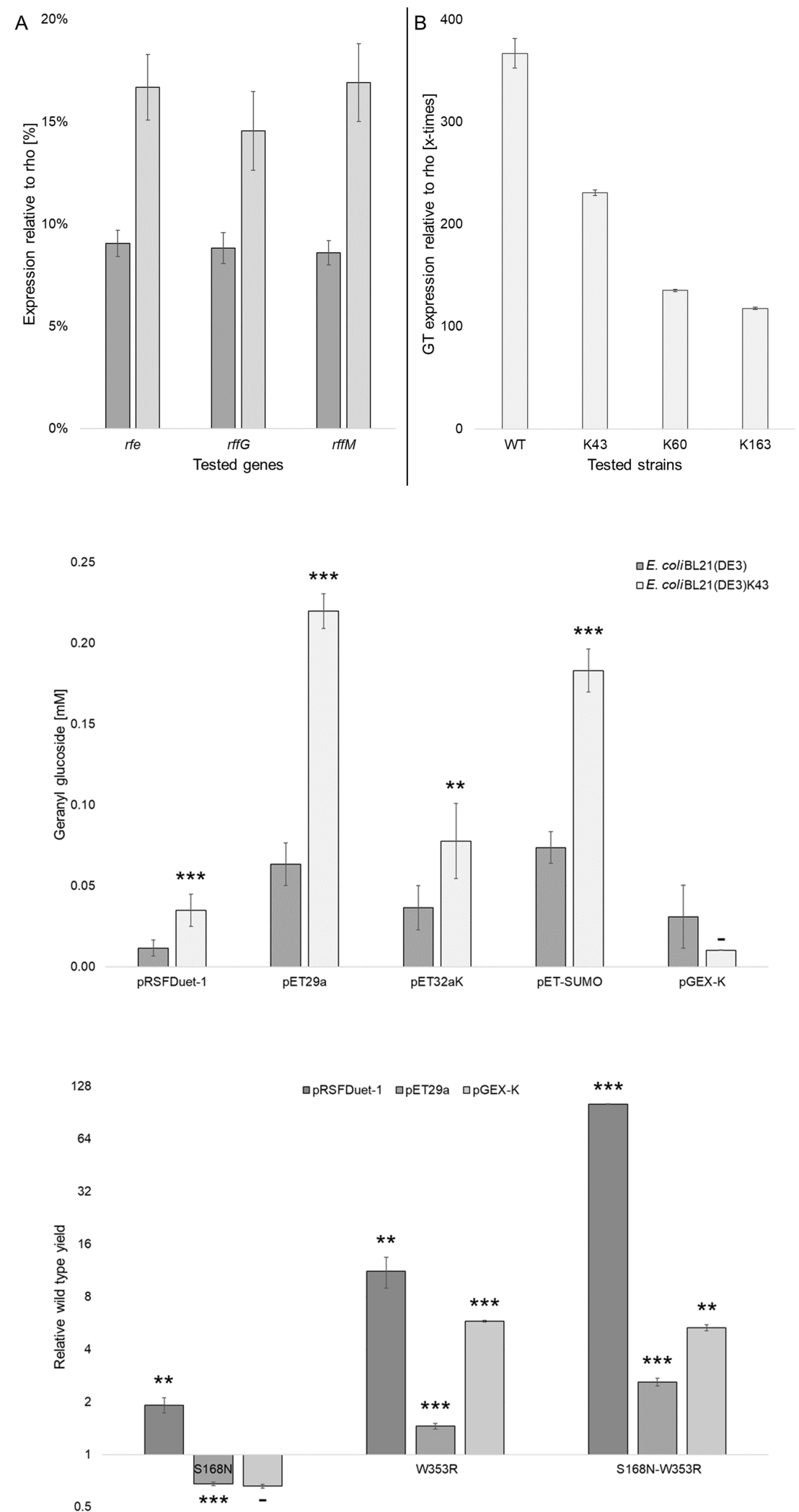

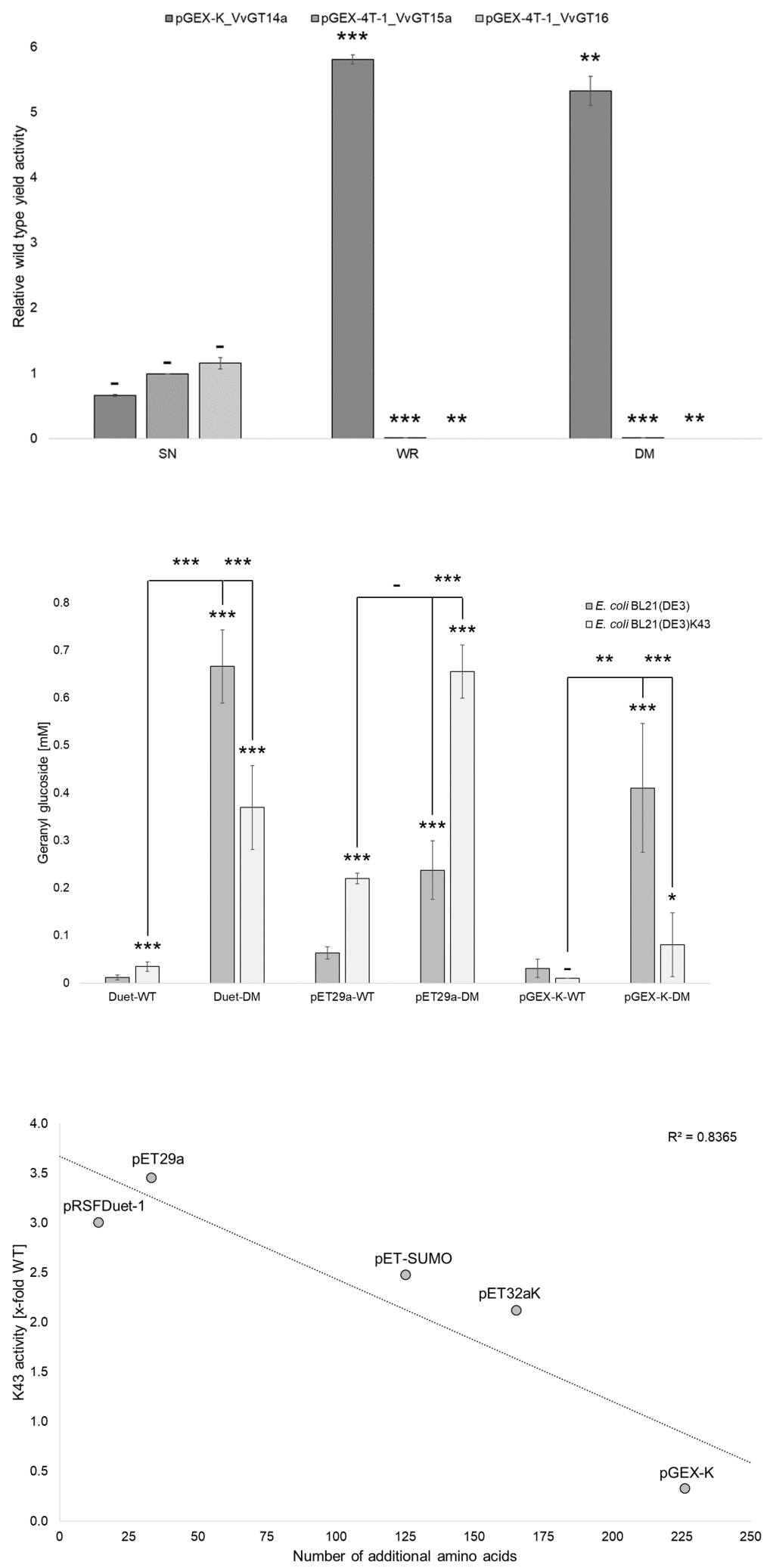